\title{
ON SUPERPOSITION OF FUNCTIONS OF BOUNDED $\varphi$-VARIATION
}

\author{
FRANCISZEK PRUS-WISNIOWSKI
}

(Communicated by Daniel Mauldin)

\begin{abstract}
J. Ciemnoczolowski and W. Orlicz in [1] have obtained some results concerning superpositions of functions of bounded $\varphi$-variation. In this note we show that the assumption in Theorem 1 of [1] that $\psi$ satisfies $\Delta_{2}$ condition may be dropped. Moreover, Theorem 2.B of [1] is extended to a stronger version.
\end{abstract}

A function $\varphi:\langle 0, \infty) \rightarrow\langle 0, \infty)$ is called a $\varphi$-function if it is continuous, nondecreasing and such that $\varphi(0)=0, \varphi(u)>0$ for $u>0$ and $\varphi(u) \rightarrow \infty$ for $u \rightarrow \infty$. A $\varphi$-function $\varphi$ satisfies condition $\Delta_{2}$ for small $u$ if $\lim \sup \varphi(2 u) /$ $\varphi(u)<\infty$ for $u \rightarrow 0+$. We denote by $X$ the vector space of real functions defined on a closed interval $\langle a, b\rangle$ which vanish at $a$. For $x \in X$, we denote by $\operatorname{osc}(x ;\langle a, b\rangle)$ the oscillation of $x$ on $\langle a, b\rangle$.

Let $\varphi$ be a $\varphi$-function and $A$ be a subset of real numbers. A finite subset $\pi$ of $A$ with the natural order we will call a partition of $A$. In general, we will write a non-empty partition $\pi$ of $A$ in form of an increasing finite sequence $\left(t_{i}\right)_{i=1}^{n}$. For a real function $x$ defined on $A$ and for a partition $\pi$ of $A$ we define

$$
\operatorname{var}_{\varphi}(x ; \pi)=\left\{\begin{array}{ll}
0 & \text { if } \operatorname{card} \pi \leq 1 \\
\sum_{i=1}^{n-1} \varphi\left(\left|x\left(t_{i+1}\right)-x\left(t_{i}\right)\right|\right) & \text { if } \operatorname{card} \pi \geq 2
\end{array} .\right.
$$

The value $\operatorname{var}_{\varphi}(x ; A)=\sup _{\pi} \operatorname{var}_{\varphi}(x ; \pi)$, where the supremum is taken over all partitions of $A$, is called a $\varphi$-variation of $x$ on $A$. If the $\varphi$-variation of $x$ is finite then we say $x$ is of bounded $\varphi$-variation. It is easy to see that if $A$ is a closed interval $\langle a, b\rangle$ then the above definition of $\varphi$-variation of $x$ on $A$ is equivalent to the classical one ([4], p. 582), which was used in [1]. The class of all functions $x \in X$ of bounded $\varphi$-variation is denoted by $V_{\varphi}\langle a, b\rangle$.

J. Ciemnoczolowski and W. Orlicz have stated in [1] the following theorem concerning superpositions of functions of bounded $\varphi$-variation.

Received by the editors May 24, 1988 and, in revised form, July 13, 1988.

1980 Mathematics Subject Classification (1985 Revision). Primary 26A45; Secondary 26A16.

Key words and phrases. Function of bounded $\varphi$-variation, composition, generalized Lipschitz condition. 
Theorem ([1], Theorem 1). Let $\varphi$ be an arbitrary $\varphi$-function, $\psi$ a $\varphi$-function satisfying $\Delta_{2}$ for small $u$. Let $F_{n}$ be real functions on $(-\infty, \infty), F_{n}(0)=0$, $n=1,2, \ldots$. Then the following are equivalent:

(a) $\sup _{n} \operatorname{var}_{\psi}\left(F_{n}(x) ;\langle a, b\rangle\right)<\infty$ for $x \in V_{\varphi}\langle a, b\rangle$;

(b) for every $r>0$ there exists a constant $C_{r}>0$ such that the inequality $\psi\left(\left|F_{n}\left(u_{1}\right)-F_{n}\left(u_{2}\right)\right|\right) \leq C_{r} \varphi\left(\left|u_{1}-u_{2}\right|\right)$ holds for $u_{1}, u_{2} \in\langle-r, r\rangle, n=1,2, \ldots$.

We will show that the assumption $\psi$ satisfies condition $\Delta_{2}$ for small $u$ may be dropped. Namely, one has

Theorem 1. Let $\left(F_{n}\right)$ be a sequence of real functions defined on $(-\infty, \infty)$ and $F_{n}(0)=0$ for $n=1,2, \ldots$. For every pair $\varphi, \psi$ of $\varphi$-functions the following statements are equivalent :

(i) For every sequence $\left(x_{n}\right)$ of functions of $X$ if $\sup _{n} \operatorname{var}_{\varphi}\left(x_{n} ;\langle a, b\rangle\right)<\infty$ then $\sup _{n} \operatorname{var}_{\psi}\left(F_{n}\left(x_{n}\right) ;\langle a, b\rangle\right)<\infty$.

(ii) If $x \in V_{\varphi}\langle a, b\rangle$ then $\sup _{n} \operatorname{var}_{\psi}\left(F_{n}(x) ;\langle a, b\rangle\right)<\infty$.

(iii) For every $r>0$ there exists a constant $C_{r}>0$ such that the inequality $\psi\left(\left|F_{n}\left(u_{1}\right)-F_{n}\left(u_{2}\right)\right|\right) \leq C_{r} \varphi\left(\left|u_{1}-u_{2}\right|\right)$ holds for $u_{1}, u_{2} \in\langle-r, r\rangle, n=$ $1,2, \ldots$.

Proof. The implication (i) $\Rightarrow$ (ii) is obvious.

(iii) $\Rightarrow$ (i). If $\sup _{n} \operatorname{var}_{\varphi}\left(x_{n} ;\langle a, b\rangle\right)$ is finite then $r=\sup _{n} \operatorname{osc}\left(x_{n} ;\langle a, b\rangle\right)$ is finite and by (iii)

$$
\sup _{n} \operatorname{var}_{\psi}\left(F_{n}\left(x_{n}\right) ;\langle a, b\rangle\right) \leq C_{r} \sup _{n} \operatorname{var}_{\varphi}\left(x_{n} ;\langle a, b\rangle\right)<\infty .
$$

(ii) $\Rightarrow$ (iii). Assume (ii) holds. Then for every $r=1,2, \ldots$ the functions $F_{n}$ are uniformly bounded in common in $\langle-r, r\rangle$ (see [1], proof of Theorem 1). If (iii) does not hold then there exist an integer $r>0$, a nondecreasing sequence $\left(n_{i}\right)$ of indices, subintervals $\left\langle u_{i}, v_{i}\right\rangle$ of $\langle-r, r\rangle$ such that

$$
d_{i}=\frac{\psi\left(\left|F_{n_{i}}\left(v_{i}\right)-F_{n_{i}}\left(u_{i}\right)\right|\right)}{\varphi\left(v_{i}-u_{i}\right)} \rightarrow \infty .
$$

Since $F_{n_{i}}$ are uniformly bounded in common in $\langle-r, r\rangle$, we have $v_{i}-u_{i} \rightarrow 0$. Without loss of generality we may assume that sequences $\left(u_{i}\right)$ and $\left(v_{i}\right)$ are convergent to a point $w$ of $\langle-r, r\rangle$. Passing, if necessary, to a partial sequence, we state that one of the following cases holds:
(A) $u_{i} \geq w$ for all $i$
(B) $v_{i} \leq w$ for all $i$;
(C) $u_{i} \leq u_{i+1}<w<v_{i+1} \leq v_{i} \quad$ for all $i$.

If (A) holds then as in ([1], proof of Theorem 1) we construct a function $x \in V_{\varphi}\langle a, b\rangle$ such that $\sup _{n} \operatorname{var}_{\psi}\left(F_{n}(x) ;\langle a, b\rangle\right)=\infty$. If (B) holds then, setting $G_{n}(u)=F_{n}(-u)$ for $u \in(-\infty, \infty)$ and $n=1,2, \ldots$, the case (A) holds for $G_{n}$. Therefore, there exists a function $x \in V_{\varphi}\langle a, b\rangle$ such that $\sup _{n} \operatorname{var}_{\psi}\left(F_{n}(x) ;\langle a, b\rangle\right)=\sup _{n} \operatorname{var}_{\psi}\left(G_{n}(x) ;\langle a, b\rangle\right)=\infty$. 
If (C) holds then there exists an increasing sequence $i_{j}$ of positive integers such that $\varphi\left(v_{i_{j}}-u_{i_{j}}\right) \leq 2^{-j-2}$ and $d_{i_{j}} \geq 2 \cdot 4^{j}$ for $j=1,2, \cdots$. We set

$$
\begin{aligned}
& s_{j}=\min \left\{s \in\{1,2, \ldots\}:(2 s-1) \varphi\left(v_{i_{j}}-u_{i_{j}}\right) \geq 2^{-j-1}\right\}, \\
& m_{0}=0, \quad m_{j}=\sum_{k=1}^{j} 2 s_{k} \quad \text { for } j=1,2, \ldots
\end{aligned}
$$

Given a decreasing sequence $\left(t_{k}\right)$ of points of $\langle a, b\rangle$ convergent to $a$ with $t_{1}=b$ and setting $x(a)=0$ and for $j=1,2, \ldots ; k=m_{j-1}+1, \ldots, m_{j}$; $t \in\left(t_{k+1}, t_{k}\right)$

$$
x(t)=\left\{\begin{array}{ll}
v_{i_{j}} & \text { for odd } k \\
u_{i_{j}} & \text { for even } k
\end{array},\right.
$$

we obtain a regulated function $x \in X$.

Now, we shall prove that $x \in V_{\varphi}\langle a, b\rangle$. By ([3], Lemma 1.1) it is enough to show that $\operatorname{var}_{\varphi}(x ;(a, b))<\infty$. Observe that for every $\varphi$-function $\chi$ and every function $F:(-\infty, \infty) \rightarrow(-\infty, \infty)$ we have

(a) $\operatorname{var}_{\chi}\left(F(x) ;\left(t_{1+m_{j}}, t_{1+m_{j-1}}\right\rangle\right)=\left(2 s_{j}-1\right) \chi\left(\left|F\left(v_{i_{j}}\right)-F\left(u_{i_{j}}\right)\right|\right)$ for $j=1,2, \ldots$,

(b) $\operatorname{var}_{\chi}\left(x ;\left(t_{1+m_{k}}, b\right\rangle\right)=\sum_{j=1}^{k} \operatorname{var}_{\chi}\left(x ;\left(t_{1+m_{j}}, t_{1+m_{j-1}}\right)\right)$

$$
+\sum_{j=1}^{k-1} \chi\left(v_{i_{j+1}}-u_{i_{j}}\right) \quad \text { for } k=1,2, \ldots
$$

Thus, for a partition $\pi=\left(r_{i}\right)_{i=1}^{n}$ of $(a, b\rangle$ if $t_{1+m_{k}}<r_{1}$ then

$$
\begin{aligned}
\operatorname{var}_{\varphi}(x ; \pi) & \leq \operatorname{var}_{\varphi}\left(x ;\left(t_{1+m_{k}}, b\right\rangle\right) \\
& \leq \sum_{j}\left(2 s_{j}-1\right) \varphi\left(v_{i_{j}}-u_{i_{j}}\right)+\sum_{j} \varphi\left(v_{i_{j+1}}-u_{i_{j}}\right) \\
& \leq \sum_{j} 2^{-j}+\sum_{j} \varphi\left(v_{i_{j}}-u_{i_{j}}\right) \leq \frac{5}{4} .
\end{aligned}
$$

Therefore $x \in V_{\varphi}\langle a, b\rangle$.

For $j=1,2, \ldots$, by (a) we have

$$
\begin{aligned}
\operatorname{var}_{\psi}\left(F_{n_{i_{j}}}(x) ;\langle a, b\rangle\right) & \geq \operatorname{var}_{\psi}\left(F_{n_{i_{j}}}(x) ;\left(t_{1+m_{j}}, t_{1+m_{j-1}}\right\rangle\right) \\
& =\left(2 s_{j}-1\right) \psi\left(\left|F_{n_{i_{j}}}\left(v_{i_{j}}\right)-F_{n_{i_{j}}}\left(u_{i_{j}}\right)\right|\right) \\
& \geq\left(2 s_{j}-1\right) \cdot 2 \cdot 4^{j} \varphi\left(v_{i_{j}}-u_{i_{j}}\right) \geq 2^{j} .
\end{aligned}
$$

Thus $\sup _{n} \operatorname{var}_{\psi}\left(F_{n}(x) ;\langle a, b\rangle\right)=\infty$ and we get a contradiction. 
If we omit the assumption that $\psi$ satisfies the $\Delta_{2}$ condition then all consequences of ([1], Theorem 1) remain true, except Theorem 2.B.

For a $\varphi$-function $\varphi$ and a real function $F$ defined on $(-\infty, \infty)$ we will write $F \in \mathrm{GL}_{\varphi}$ if $F(0)=0$ and $F$ satisfies the following generalized Lipschitz condition: for every $k>0$ there exists a constant $C_{k}>0$ such that $\varphi(\mid F(u)-$ $F(v) \mid) \leq C_{k} \varphi(|u-v|)$ for $u, v \in\langle-k, k\rangle$. It is easy to see that functions from $\mathrm{GL}_{\varphi}$ are continuous. If $\varphi(u)=u$ then we will write $\mathrm{GL}$ instead $\mathrm{GL}_{\varphi}$. Using this notation the Theorem 2.A of [1] may be written as follows: $F\left(V_{\varphi}\langle a, b\rangle\right) \subset$ $V_{\varphi}\langle a, b\rangle$ iff $F \in \mathrm{GL}_{\varphi}$.

J. Ciemnoczolowski and W. Orlicz have formulated in [1] a sufficient condition for the equality $\mathrm{GL}_{\varphi}=\mathrm{GL}$. Namely, they have proved the following

Theorem ([1], Theorem 2.B). Let $\varphi$ be a strictly increasing $\varphi$-function such that $\varphi$ and $\varphi^{-1}$ satisfy condition $\Delta_{2}$ for small $u$. Then $\mathrm{GL}_{\varphi}=\mathrm{GL}$.

Theorems 2 and 3 jointly allow to formulate the necessary and sufficient condition for the equality $\mathrm{GL}_{\varphi}=\mathrm{GL}$.

Theorem 2. The inclusion $\mathrm{GL}_{\varphi} \subset \mathrm{GL}$ holds if and only if $\varphi$ satisfies the condition

(E) for every $c>0$ there exists a number $r>0$ such that

$$
\limsup _{u \rightarrow 0^{+}} \frac{\varphi(r u)}{\varphi(u)}>c \text {. }
$$

To prove this theorem we need a simple lemma.

Lemma. Let $F$ be a real function defined on $\langle a, b\rangle$. Then for every positive integer $n$ there exist points $s, t$ of $\langle a, b\rangle$ such that

$$
t-s=\frac{b-a}{n}
$$

and

$$
\frac{|F(t)-F(s)|}{t-s} \geq \frac{|F(b)-F(a)|}{b-a} .
$$

Proof. If for some integer $n>0$ and every pair $s, t$ of points of $\langle a, b\rangle$, satisfying (1), the inequality

$$
\frac{|F(t)-F(s)|}{t-s}<\frac{|F(b)-F(a)|}{b-a}
$$

holds, then setting $s_{k}=a+(k-1)(b-a) / n$ and $t_{k}=a+k(b-a) / n$ for $k=1, \ldots, n$, we have

$$
\begin{aligned}
|F(b)-F(a)| & \leq \sum_{k=1}^{n}\left|F\left(t_{k}\right)-F\left(s_{k}\right)\right| \\
& <\frac{|F(b)-F(a)|}{b-a} \sum_{k=1}^{n}\left(t_{k}-s_{k}\right)=|F(b)-F(a)|
\end{aligned}
$$

and we get a contradiction. 
Proof of Theorem 2. First, suppose that $\varphi$ satisfies the condition (E) and that $F \in \mathrm{GL}_{\varphi} \backslash \mathrm{GL}$. Let $m$ be a positive number such that for every $k>0$ there exists a subinterval $\left\langle u_{k}, v_{k}\right\rangle$ of $\langle-m, m\rangle$ such that $\left|F\left(v_{k}\right)-F\left(u_{k}\right)\right| \geq$ $k\left(v_{k}-u_{k}\right)$. Let $C>0$ be such that $\varphi(|F(u)-F(v)|) \leq C \varphi(|u-v|)$ for $u, v \in\langle-m, m\rangle$.

For some $r>0$ we have

$$
\limsup _{u \rightarrow 0^{+}} \frac{\varphi(r u)}{\varphi(u)}>C+1
$$

and there exists a subinterval $\langle u, v\rangle$ of $\langle-m, m\rangle$ such that $|F(v)-F(u)| \geq$ $(r+1)(v-u)$. Since $F$ is continuous, it follows that there exists an $\varepsilon \in(0, v-u)$ such that

$$
|F(v)-F(s)| \geq r(v-s) \quad \text { for } s \in\langle u, u+\varepsilon\rangle .
$$

Choosing a number $w \in(0, \varepsilon)$ so that

$$
\varphi(r w)>(C+1) \varphi(w),
$$

we have $v-l w \in\langle u, u+\varepsilon\rangle$ for some integer $l>0$. Thus, by $(+) \mid F(v)-F(v-$ $\mid w) \mid \geq r l w$ and therefore, by our Lemma there exists a subinterval $\left\langle u^{\prime}, v^{\prime}\right\rangle$ of $\langle v-l w, v\rangle$ such that $v^{\prime}-u^{\prime}=w$ and $\left|F\left(v^{\prime}\right)-F\left(u^{\prime}\right)\right| \geq r\left(v^{\prime}-u^{\prime}\right)$. Thus,

$$
\begin{aligned}
\varphi(r w)=\varphi\left(r\left(v^{\prime}-u^{\prime}\right)\right) & \leq \varphi\left(\left|F\left(v^{\prime}\right)-F\left(u^{\prime}\right)\right|\right) \\
& \leq C \varphi\left(v^{\prime}-u^{\prime}\right)<(C+1) \varphi(w),
\end{aligned}
$$

which contradicts (++). So if $\varphi$ satisfies the condition (E) then $\mathrm{GL}_{\varphi} \subset \mathrm{GL}$.

Conversely, suppose that $\varphi$ does not satisfy (E). Then there exists a constant $c>1$ such that for every $n=1,2, \ldots$ there exists a number $v_{n}>0$ such that $\varphi((n+1) u) \leq c \varphi(u)$ for $u \in\left(0, v_{n}\right)$. For a sequence $\left(u_{n}\right)$ of positive numbers such that $u_{1} \leq 1, u_{n}<v_{n}$ and $2(n+1) u_{n+1}<n u_{n}$ for $n=1,2, \ldots$, the series $\sum_{n}(-1)^{n} n u_{n}$ is convergent. Now, we define a real function on $(-\infty, \infty)$, setting

$$
\begin{aligned}
t_{n} & =-\sum_{i=n}^{\infty} u_{i} \quad \text { for } n=1,2, \ldots ; \\
F(t)=0 \text { for } t & \geq 0 ; \quad F(t)=\sum_{n}(-1)^{n} n u_{n} \quad \text { for } t<t_{1} ; \\
F\left(t_{n}\right) & =\sum_{i=n}^{\infty}(-1)^{i} i u_{i} \quad \text { for } n=1,2, \ldots
\end{aligned}
$$

Finally, we define $F$ to be a linear function on each $\left\langle t_{n}, t_{n+1}\right\rangle$. Observe that for every integer $n>0$ and every $u \in\left\langle u_{n+1}, u_{n}\right)$ we have $|F(d+u)-F(d)| \leq$ $(n+1) u_{n+1}$ for $d \geq t_{n+1}$ and $|F(d+u)-F(d)| \leq n u$ for $d<t_{n+1}$. Hence for every real number $d$

$$
|F(d+u)-F(d)| \leq(n+1) u \quad \text { for } u \subset\left\langle u_{n+1}, u_{n}\right) .
$$


More, observe that for $u \geq u_{1}$ and for every real number $d$ $(++++)$

$$
|F(d+u)-F(d)| \leq u_{1} .
$$

Given two different real numbers $w, w^{\prime}$, if $\left|w-w^{\prime}\right| \geq u_{1}$ then by $(++++) \varphi\left(F(w)-F\left(w^{\prime}\right) \mid\right) \leq \varphi\left(u_{1}\right) \leq c \varphi\left(\left|w-w^{\prime}\right|\right)$. If $\left|w-w^{\prime}\right| \in\left\langle u_{n+1}, u_{n}\right)$ then by $(+++)$

$$
\varphi\left(\left|F(w)-F\left(w^{\prime}\right)\right|\right) \leq \varphi\left((n+1)\left|w-w^{\prime}\right|\right) \leq c \varphi\left(\left|w-w^{\prime}\right|\right),
$$

because $u_{n}<v_{n}$. We have proved that $F \in \mathrm{GL}_{\varphi}$. Finally, for $n=1,2, \ldots$ we have $t_{n} \in\langle-2,2\rangle$ and

$$
\frac{\left|F\left(t_{n+1}\right)-F\left(t_{n}\right)\right|}{\left|t_{n+1}-t_{n}\right|}=\frac{n u_{n}}{u_{n}}=n .
$$

Thus $F \notin \mathrm{GL}$. So if $\mathrm{GL}_{\varphi} \subset \mathrm{GL}$ then $\varphi$ satisfies the condition (E).

Theorem 3. The inclusion $\mathrm{GL} \subset \mathrm{GL}_{\varphi}$ holds if and only if $\varphi$ satisfies the condition $\Delta_{2}$ for small $u$.

Proof. First, assume that $\varphi$ satisfies $\Delta_{2}$ for small $u$ and $F \in \mathrm{GL}$. Given $m>0$, there exists a constant $C>0$ such that $\varphi(|F(u)-F(v)|) \leq \varphi(C|u-v|)$ for $u, v \in\langle-m, m\rangle$. Because $\varphi$ satisfies $\Delta_{2}$ for small $u$, there exists a constant $K_{m}>0$ such that $\varphi(C w) \leq K_{m} \varphi(w)$ for $0 \leq w \leq 2 m$ (cf. [2], 1.02). Thus, $\varphi(|F(u)-F(v)|) \leq K_{m} \varphi(|u-v|)$ for $u, v \in\langle-m, m\rangle$. It follows that $F \in \mathrm{GL}_{\varphi}$. Then

Conversely, suppose that $\varphi$ does not satisfy the condition $\Delta_{2}$ for small $u$.

$$
\limsup _{u \rightarrow 0^{+}} \frac{\varphi(2 u)}{\varphi(u)}=\infty \text {. }
$$

and it is easy to see that for $F(u)=2 u$ we have $F \in \mathrm{GL}$ and $F \notin \mathrm{GL}_{\varphi}$.

The following result is just exactly a generalization of Theorem 2.B of [1].

Corollary. The identity $\mathrm{GL}=\mathrm{GL}_{\varphi}$ holds if and only if $\varphi$ satisfies the conditions (E) and $\Delta_{2}$ for small $u$.

\section{REFERENCES}

1. J. Ciemnoczolowski and W. Orlicz, Composing functions of bounded $\varphi$-variation, Proc. Amer. Math. Soc. 96 (1986), 431-436.

2. J. Musielak and W. Orlicz, On generalized variations. I, Studia Math. 18 (1959), 11-41.

3. F. Prus-Wiśniowski, Some remarks on functions of bounded $\varphi$-variation, Comment. Math. 30 (1991) (to appear).

4. L. C. Young, General inequalities for Stieltjes integrals and the convergence of Fourier series, Math. Ann. 115 (1938), 581-612.

Institute of Mathematics, University of Szczecin, Ul. Wielkopolska 15, 70-451 Szczecin, Poland 